\title{
Further Results on Colombeau Product of Distributions
}

\author{
Biljana Jolevska-Tuneska ${ }^{1}$ and Tatjana Atanasova-Pacemska ${ }^{2}$ \\ ${ }^{1}$ Faculty of Electrical Engineering and Informational Technologies, Saints Cyril and Methodius University of Skopje, \\ Karpos II bb, 1000 Skopje, Macedonia \\ ${ }^{2}$ Faculty of Computer Sciences, Goce Delčev University of Štip, Krste Misirkov, Br 10A, 2000 Štip, Macedonia
}

Correspondence should be addressed to Biljana Jolevska-Tuneska; biljanaj@feit.ukim.edu.mk

Received 10 August 2012; Revised 28 November 2012; Accepted 10 December 2012

Academic Editor: Shyam Kalla

Copyright (C) 2013 B. Jolevska-Tuneska and T. Atanasova-Pacemska. This is an open access article distributed under the Creative Commons Attribution License, which permits unrestricted use, distribution, and reproduction in any medium, provided the original work is properly cited.

Results on Colombeau product of distributions $x_{+}^{-r-1 / 2}$ and $x_{-}^{-r-1 / 2}$ are derived. They are obtained in Colombeau differential algebra $\mathscr{G}(\mathbf{R})$ of generalized functions that contains the space $\mathscr{D}^{\prime}(\mathbf{R})$ of Schwartz distributions as a subspace and has a notion of "association" that is a faithful generalization of the weak equality in $\mathscr{D}^{\prime}(\mathbf{R})$.

\section{Introduction}

In quantum physics one finds the need to evaluate $\delta^{2}$, when calculating the transition rates of certain particle interactions; see [1]. The problem of defining products of distributions is also closely connected with the problem of renormalization in quantum field theory. Due to the large use of distributions in the natural sciences and other mathematical fields, the problem of the product of distributions [2] is an objective of many research studies. Starting with the historically first construction of distributional multiplication by König [3], and the sequential approach developed in [4] by Antosik et al., there have been numerous attempts to define products of distributions, see [5-7], or rather to enlarge the number of existing products.

Having a look at the theory of distributions [8, 9] we realize that there are two complementary points of view.

(1) A distribution $f \in \mathscr{D}^{\prime}\left(\mathbf{R}^{n}\right)$ is a continuous functional on the space $\mathscr{D}\left(\mathbf{R}^{n}\right)$ of space functions (compactly supported smooth functions). Here we have a linear action

$$
\varphi \longrightarrow\langle f, \varphi\rangle
$$

of $f$ on a test function $\phi$.
(2) Letting $\left\{\varphi_{n}\right\}$ be a sequence of smooth functions converging to the Dirac measure, a family of regularization $\left\{f_{n}\right\}$ can be produced by convolution,

$$
f_{n}(x)=f * \varphi_{n}=\left\langle f(y), \varphi_{n}(x-y)\right\rangle,
$$

which converges weakly to the original distribution $f \in \mathscr{D}^{\prime}\left(\mathbf{R}^{n}\right)$. Identifying two sequences $\left\{\varphi_{n}\right\}$ and $\{\bar{\varphi}\}_{n}$ if they have the same limit, we obtain a sequential representation of the space of distributions. Other authors use the equivalence classes of nets of regularization. The delta-net $\left\{\varphi_{\varepsilon}\right\}_{\varepsilon>0}$ is defined by

$$
\varphi_{\varepsilon}(x)=\varepsilon^{-n} \varphi\left(\frac{x}{\varepsilon}\right) .
$$

When we work with regularization the nonlinear structure is lost by identifying sequences (nets) with the same limit. So we have to get nonlinear theory of generalized functions that will work with regularization, but identify less. The actual construction of such algebras enjoying these optimal properties is due to Colombeau [10]. His theory of algebras of generalized functions offers the possibility of applying large classes of nonlinear operations to distributional objects. Some of them are used on solving differential equations with nonstandard coefficients [11]. The "association process" in 
Colombeau algebra providing a faithful generalization of the equality of distributions in $\mathscr{D}\left(\mathbf{R}^{n}\right)$ enables us to obtain results in terms of distributions, the so-called Colombeau products. In fact, we evaluate particular product of distributions with coinciding singularities, as embedded in Colombeau algebra, in terms of associated distributions; see [12-14]. Therefore, the results obtained can be reformulated as regularized model products in the classical distribution theory.

In 1966, Mikusinski published his famous result [15]:

$$
x^{-1} \cdot x^{-1}-\pi^{2} \delta(x) \delta(x)=x^{-2}
$$

where neither of the products on the left-hand side here exists, but their difference still has a correct meaning in distribution space $\mathscr{D}^{\prime}(\mathbf{R})$. Formula including balanced products of distributions with coinciding singularities can be found in mathematical and physical literature. In this paper results on product of distributions $x_{+}^{-r-1 / 2}$ and $x_{-}^{-r-1 / 2}$ are derived.

\section{Colombeau Algebra}

The basic idea underlying Colombeau's theory in its simplest form is that of embedding the space of distributions into a factor algebra of $\mathscr{C}^{\infty}\left(\mathbf{R}^{n}\right)^{I}, I=(0,1)$ with regularization by convolution with a fixed "mollifier" $\varphi$.

The space of test function is

$$
\mathscr{A}_{0}\left(\mathbf{R}^{n}\right)=\left\{\varphi \in \mathscr{D}\left(\mathbf{R}^{n}\right): \int \varphi(x) d x=1\right\} .
$$

Functional act on test functions and points is

$$
\begin{gathered}
u: \mathscr{A}_{0}\left(\mathbf{R}^{n}\right) \times \mathbf{R}^{n} \longrightarrow \mathbf{C}, \\
(\varphi, x) \longrightarrow u(\varphi, x),
\end{gathered}
$$

where $u(\varphi, x)$ is required to belong to $\mathscr{C}^{\infty}\left(\mathbf{R}^{n}\right)$ with respect to the second variable $x \in \mathbf{R}^{n}$. Now let $\varphi_{\varepsilon}(x)=$ $\varepsilon^{-n} \varphi(x / \varepsilon)$ for $\varphi \in \mathscr{A}_{0}\left(\mathbf{R}^{n}\right)$. The sequence $\left(u * \varphi_{\varepsilon}\right)_{\varepsilon \in I}$ converges to $u$ in $\mathscr{D}^{\prime}\left(\mathbf{R}^{n}\right)$. Taking this sequence as a representative of $u$ we obtain an embedding of $\mathscr{D}^{\prime}\left(\mathbf{R}^{n}\right)$ into the algebra $\mathscr{C}^{\infty}\left(\mathbf{R}^{n}\right)$. However, embedding $\mathscr{C}^{\infty}\left(\mathbf{R}^{n}\right) \subset \mathscr{D}^{\prime}\left(\mathbf{R}^{n}\right)$ into this algebra via convolution as above will not yield a subalgebra since of course $\left(f * \varphi_{\varepsilon}\right)\left(g * \varphi_{\varepsilon}\right) \neq(f g) * \varphi_{\varepsilon}$ in general. The idea, therefore, is to factor out an ideal $\mathscr{N}\left(\mathbf{R}^{n}\right)$ such that this difference vanishes in the resulting quotient. In order to construct $\mathcal{N}\left(\mathbf{R}^{n}\right)$ it is obviously sufficient to find an ideal containing all differences $\left(f * \varphi_{\varepsilon}\right)-(f)_{\varepsilon \in I}$. Taylor expansion of $\left(f * \varphi_{\varepsilon}\right)-f$ shows that this term will vanish faster than any power of $\varepsilon$, uniformly on compact sets, in all derivatives. We use the following space:

$$
\begin{aligned}
& \mathscr{A}_{q}\left(\mathbf{R}^{n}\right) \\
& \quad=\left\{\varphi \in \mathscr{A}_{0}\left(\mathbf{R}^{n}\right): \int x^{\alpha} \varphi(x) d x=0 \text { for } 1 \leq|\alpha| \leq q\right\} ;
\end{aligned}
$$

moderate functionals, denoted by $\mathscr{E}_{M}\left(\mathbf{R}^{n}\right)$, are defined by the property:

$$
\begin{gathered}
\forall K \subset \subset \mathbf{R}^{n} \forall \alpha \in \mathbf{N}_{0}^{n} \exists p \geq 0 \quad \text { such that } \forall \varphi \in \mathscr{A}_{q}\left(\mathbf{R}^{n}\right), \\
\sup _{x \in K}\left|\partial^{\alpha} u\left(\varphi_{\varepsilon}, x\right)\right|=\mathcal{O}\left(\varepsilon^{-p}\right) \quad \text { as } \varepsilon \longrightarrow 0 .
\end{gathered}
$$

Null functionals, denoted by $\mathcal{N}\left(\mathbf{R}^{n}\right)$, are defined by the property:

$$
\begin{aligned}
& \forall K \subset \subset \mathbf{R}^{n} \forall \alpha \in \mathbf{N}_{0}^{n} \exists p \geq 0 \\
& \text { such that } \forall q \geq p, \forall \varphi \in \mathscr{A}_{q}\left(\mathbf{R}^{n}\right), \\
& \sup _{x \in K}\left|\partial^{\alpha} u\left(\varphi_{\varepsilon}, x\right)\right|=\mathcal{O}\left(\varepsilon^{q-p}\right) \quad \text { as } \varepsilon \longrightarrow 0 .
\end{aligned}
$$

In other words, moderate functionals satisfy a locally uniform polynomial estimate as $\varepsilon \rightarrow 0$ when acting on $\varphi_{\varepsilon}$, together with all derivatives, while null functionals vanish faster than any power of $\varepsilon$ in the same situation. The null functionals form a differential ideal in the collection of moderate functionals.

We define space of functions $u: \mathscr{A}_{0} \rightarrow \mathscr{C}$ and denote by $\mathscr{E}_{0}\left(\mathbf{R}^{n}\right)$. It is a subalgebra in $\mathscr{E}\left(\mathbf{R}^{n}\right)$ in sense of natural identification.

Moderate functionals, denoted by $\mathscr{E}_{0 M}\left(\mathbf{R}^{n}\right)$, are defined by the property:

$$
\begin{gathered}
\exists p \geq 0 \quad \text { such that } \forall \varphi \in \mathscr{A}_{p}\left(\mathbf{R}^{n}\right), \\
\left|u\left(\varphi_{\varepsilon}\right)\right|=\mathcal{O}\left(\varepsilon^{-p}\right) \quad \text { as } \varepsilon \longrightarrow 0 .
\end{gathered}
$$

Null functionals, denoted by $\mathcal{N}_{0}\left(\mathbf{R}^{n}\right)$, are defined by the property:

$$
\begin{gathered}
\exists p \geq 0 \quad \text { such that } \forall q \geq p, \forall \varphi \in \mathscr{A}_{q}\left(\mathbf{R}^{n}\right), \\
\left|u\left(\varphi_{\varepsilon}\right)\right|=\mathcal{O}\left(\varepsilon^{q-p}\right) \quad \text { as } \varepsilon \longrightarrow 0 .
\end{gathered}
$$

Definition 1. Space of generalized functions $\mathscr{G}\left(\mathbf{R}^{n}\right)$, generalized complex numbers, and generalized real numbers is the factor algebra defined as

$$
\mathscr{G}\left(\mathbf{R}^{n}\right)=\frac{\mathscr{E}_{M}\left(\mathbf{R}^{n}\right)}{\mathcal{N}\left(\mathbf{R}^{n}\right)}, \quad \overline{\mathbf{C}}=\frac{\mathscr{E}_{0 M}(\mathbf{C})}{\mathcal{N}_{0}(\mathbf{C})}, \quad \overline{\mathbf{R}}=\frac{\mathscr{E}_{0 M}(\mathbf{R})}{\mathcal{N}_{0}(\mathbf{R})}
$$

The space of distributions is imbedded by convolution:

$$
\begin{gathered}
i: \mathscr{D}^{\prime}\left(\mathbf{R}^{n}\right) \longrightarrow \mathscr{G}\left(\mathbf{R}^{n}\right), \\
i(u)=\text { class of }[(\varphi, x) \longrightarrow u * \varphi(x)] .
\end{gathered}
$$

Equivalence classes of sequences $\left(u_{\varepsilon}\right)_{\varepsilon \in I}$ in $\mathscr{G}\left(\mathbf{R}^{n}\right)$ will be denoted by $U=$ class $\left[\left(u_{\varepsilon}\right)_{\varepsilon \in I}\right]$. If $G$ is a generalized function with compact support $K \subset \subset \mathbf{R}^{n}$ and $G_{\varepsilon}(x)$ is a representative of $G$, then its integral is defined by

$$
\int G d x=\text { class }\left[\int \varphi(x) G_{\varepsilon}(x) d x\right] .
$$

Let $F, G \in \mathscr{G}\left(\mathscr{R}^{n}\right)$. Then 
(i) they are equal in the distribution sense, $G={ }^{\mathscr{D}^{\prime}} F$ if

$$
\int(G-F) \varphi d x=0 \in \overline{\mathscr{C}}
$$

for any $\varphi \in \mathscr{D}\left(\mathbf{R}^{n}\right)$;

(ii) they are associated $G \approx F$ if there exist a representative $G_{\varepsilon}$ and $F_{\varepsilon}$ of $G$ and $F$, respectively, such that

$$
\lim _{\varepsilon \rightarrow 0} \int\left(G_{\varepsilon}(x)-F_{\varepsilon}(x)\right) \varphi(x) d x=0,
$$

for any $\varphi \in \mathscr{D}\left(\mathbf{R}^{n}\right)$.

\section{Results on Some Products of Distributions}

It was proved in [12] that for any $a \in \mathbf{R} / \mathbf{Z}$ the product of the generalized functions $\widetilde{x_{+}^{a}}$ and $\widetilde{x_{-}^{-a-1}}$ in $\mathscr{G}(\mathbf{R})$ admits associated distributions and it holds

$$
\begin{aligned}
\widetilde{x_{+}^{a}} \cdot \widetilde{x_{-}^{-a-1}}=\widetilde{x_{-}^{-a-1}} \cdot \widetilde{x_{+}^{a}} & \approx \frac{\Gamma(1+a) \Gamma(-a)}{2} \delta(x) \\
& =-\frac{\pi}{2} \csc (\pi a) \delta(x),
\end{aligned}
$$

with the particular cases

$$
\widetilde{x_{+}^{-r-1 / 2}} \cdot \widetilde{x_{-}^{r-1 / 2}}=\frac{(-1)^{r} \pi}{2} \delta(x),
$$

for $r=0, \pm 1, \pm 2, \ldots$ and

$$
\widetilde{x_{+}^{-1 / 2}} \cdot \widetilde{x_{-}^{-1 / 2}}=\frac{\pi}{2} \delta(x) \text {. }
$$

Here we will make a generalization of (19). In order to prove the main theorem we need the following lemmas, easily proved by induction.

Lemma 2. For $p<r$ one has

$$
\begin{aligned}
\int_{t}^{d} s^{p} \varphi^{(r)}(s) d s= & \sum_{k=0}^{p}(-1)^{k} \frac{p !}{(p-k) !} t^{p-k} \varphi^{(r-k-1)} \\
& \times(t) d t, \quad \text { for } \mathrm{p}<\mathrm{r} .
\end{aligned}
$$

Lemma 3. For $p=r$ one has

$$
\int_{t}^{d} s^{r} \varphi^{(r)}(s) d s=\sum_{k=0}^{r}(-1)^{k} \frac{r !}{(r-k) !} t^{r-k} \varphi^{(r-k-1)}(t) d t
$$

and $\varphi^{(-1)}(t)$ stands for $\int_{t}^{d} \varphi(s) d s$.

Theorem 4. The product of the generalized functions $\widetilde{x_{+}^{-r-1 / 2}}$ and $\widetilde{x_{-}^{-r-1 / 2}}$ for $r=0,1,2, \ldots$ in $\mathscr{G}(\mathbf{R})$ admits associated distributions and it holds

$$
\widetilde{x_{+}^{-r-1 / 2}} \cdot \widetilde{x_{-}^{-r-1 / 2}} \approx \frac{\pi}{2(2 r) !} \delta^{(2 r)}(x) .
$$

Proof. For given $\varphi \in A_{0}(\mathbf{R})$ we suppose that $\operatorname{supp} \varphi(x) \subseteq$ $[c, d]$, without loss of generality. Then using the embedding rule and the substitution $t=(y-x) / \varepsilon$ we have the representatives of the distribution $x_{+}^{-r-1 / 2}$ in Colombeau algebra:

$$
\begin{aligned}
\widetilde{x_{+}^{-r-1 / 2}}\left(\varphi_{\varepsilon}, x\right) & =(-1)^{r} \frac{2^{r}}{(2 r-1) ! !} \frac{1}{\varepsilon} \frac{\partial^{r}}{\partial x} \int_{0}^{\infty} y^{-1 / 2} \varphi\left(\frac{y-x}{\varepsilon}\right) d y \\
& =\frac{2^{r}}{(2 r-1) ! !} \frac{1}{\varepsilon^{r+1}} \int_{0}^{\infty} y^{-1 / 2} \varphi^{(r)}\left(\frac{y-x}{\varepsilon}\right) d y \\
& =\frac{2^{r}}{(2 r-1) ! !} \frac{1}{\varepsilon^{r}} \int_{-x / \varepsilon}^{d}(x+\varepsilon t)^{-1 / 2} \varphi^{(r)}(t) d t .
\end{aligned}
$$

Similar, using the embedding rule and the substitution $s=(y-x) / \varepsilon$ we have the representatives of the distribution $x_{-}^{-r-1 / 2}$ in Colombeau algebra:

$$
\begin{aligned}
\widetilde{x_{-}^{-r-1 / 2}}\left(\varphi_{\varepsilon}, x\right)= & (-1)^{r} \frac{2^{r}}{(2 r-1) ! !} \frac{1}{\varepsilon} \frac{\partial^{r}}{\partial x} \\
& \times \int_{-\infty}^{0}(-y)^{-1 / 2} \varphi\left(\frac{y-x}{\varepsilon}\right) d y \\
= & \frac{2^{r}}{(2 r-1) ! !} \frac{1}{\varepsilon^{r+1}} \\
& \times \int_{-\infty}^{0}(-y)^{-1 / 2} \varphi^{(r)}\left(\frac{y-x}{\varepsilon}\right) d y \\
= & \frac{2^{r}}{(2 r-1) ! !} \frac{1}{\varepsilon^{r}} \int_{c}^{-x / \varepsilon}(-x-\varepsilon s)^{-1 / 2} \varphi^{(r)}(s) d s .
\end{aligned}
$$

Then, for any $\psi(x) \in \mathscr{D}(\mathbf{R})$ we have

$$
\begin{aligned}
&\left\langle\widetilde{x_{+}^{-r-1 / 2}}\left(\varphi_{\varepsilon}, x\right) \cdot \widetilde{x_{-}^{-r-1 / 2}}\left(\varphi_{\varepsilon}, x\right), \psi(x)\right\rangle \\
&=\int_{-\infty}^{\infty} \widetilde{x_{+}^{-r-1 / 2}}\left(\varphi_{\varepsilon}, x\right) \widetilde{x_{-}^{-r-1 / 2}}\left(\varphi_{\varepsilon}, x\right) \psi(x) d x \\
&= \frac{2^{2 r}}{((2 r-1) ! !)^{2}} \frac{1}{\varepsilon^{2 r}} \int_{-d \varepsilon}^{c \varepsilon} \psi(x) \int_{-x / \varepsilon}^{d} \varphi^{(r)}(t) \\
& \times \int_{c}^{-x / \varepsilon}(x+\varepsilon t)^{-1 / 2}(-x-\varepsilon s)^{-1 / 2} \varphi^{(r)}(s) d s d t d x \\
&= \frac{2^{2 r}}{((2 r-1) ! !)^{2}} \frac{1}{\varepsilon^{2 r}} \int_{c}^{d} \psi(-\varepsilon \omega) \int_{\omega}^{d} \varphi^{(r)}(t) \\
& \times \int_{c}^{\omega}(t-\omega)^{-1 / 2}(\omega-s)^{-1 / 2} \varphi^{(r)}(s) d s d t d \omega,
\end{aligned}
$$

using the substitution $\omega=-x / \varepsilon$.

By the Taylor theorem we have that

$$
\psi(-\varepsilon \omega)=\sum_{k=0}^{2 r} \frac{\psi^{(k)}(0)}{k !}(-\varepsilon \omega)^{k}+\frac{\psi^{(2 r+1)}(\eta \omega)}{(2 r+1) !}(-\varepsilon \eta)^{2 r+1},
$$


for $\eta \in(0,1)$. Using this for $(25)$ we have

$$
\begin{aligned}
& \left\langle\widetilde{x_{+}^{-r-1 / 2}}\left(\varphi_{\varepsilon}, x\right) \cdot \widetilde{x_{-}^{-r-1 / 2}}\left(\varphi_{\varepsilon}, x\right), \psi(x)\right\rangle \\
& =\frac{2^{2 r}}{((2 r-1) ! !)^{2}} \sum_{k=0}^{2 r} \frac{(-1)^{k} \psi^{(k)}(0)}{k ! \varepsilon^{2 r-k}} I_{k}+O(\varepsilon),
\end{aligned}
$$

where

$$
\begin{aligned}
I_{k}= & \int_{c}^{d} \varphi^{(r)}(t) \int_{t}^{d} \varphi^{(r)}(s) \\
& \times \int_{s}^{t}(t-\omega)^{-1 / 2}(\omega-s)^{-1 / 2} \omega^{k} d \omega d s d t
\end{aligned}
$$

for $k=0,1, \ldots, 2 r$ and we have changed the order of integration.

Putting $\omega-s=(t-s) v$ we have

$$
\begin{aligned}
\int_{s}^{t}(t & -\omega)^{-1 / 2}(\omega-s)^{-1 / 2} \omega^{k} d \omega \\
& =\int_{0}^{1} v^{-1 / 2}(1-v)^{-1 / 2}[s v+(1-v) t]^{k} d v \\
& =\sum_{p=0}^{k} \frac{k !}{p !(k-p) !} \int_{0}^{1} v^{p-1 / 2}(1-v)^{k-p-1 / 2} s^{p} t^{k-p} d v \\
& =\sum_{p=0}^{k} \frac{k !}{p !(k-p) !} B\left(p+\frac{1}{2}, k-p+\frac{1}{2}\right) s^{p} t^{k-p} .
\end{aligned}
$$

Thus

$$
\begin{aligned}
I_{k}= & \sum_{p=0}^{k} \frac{k !}{p !(k-p) !} B\left(p+\frac{1}{2}, k-p+\frac{1}{2}\right) \\
& \times \int_{c}^{d} t^{k-p} \varphi^{(r)}(t) \int_{t}^{d} s^{p} \varphi^{(r)}(s) d s d t \\
= & \sum_{p=0}^{k} \frac{k !}{p !(k-p) !} B\left(p+\frac{1}{2}, k-p+\frac{1}{2}\right) J_{k, p} .
\end{aligned}
$$

Next, suppose that $k$ is even, less than $2 r$ and that $p$ is less than $r$. It follows from Lemma 2 that $\int_{t}^{d} s^{p} \varphi^{(r)}(s) d s$ is an even or odd function accordingly as $r+p$ is odd or even. We thus have that $t^{k-p} \varphi^{(r)}(t) \int_{t}^{d} s^{p} \varphi^{(r)}(s) d s$ is an odd function and $J_{k, p}=0$. If $p \geq r$ then $k-p<r$ and by changing the order of integration we can prove that again $J_{k, p}=0$. If we suppose that $k$ is odd and less than $2 r$ we can prove in a similar manner that $J_{k, p}=0$.
For the case $k=2 r$ if $p \neq r$ again we have $J_{k, p}=0$. For $k=2 r$ and $p=r$ using Lemma 3 and changing the order of integration we have

$$
\begin{aligned}
J_{2 r, r} & =\int_{c}^{d} t^{r} \varphi^{(r)}(t) \int_{t}^{d} s^{r} \varphi^{(r)}(s) d s d t \\
& =\sum_{i=0}^{r} \frac{(-1)^{i} r !}{(r-i) !} \int_{c}^{d} t^{2 r-i} \varphi^{(r)}(t) \varphi^{(r-i-1)}(t) d t \\
& =(-1)^{r} r ! \int_{c}^{d} t^{r} \varphi^{(r)}(t) \int_{t}^{d} \varphi(s) d s d t \\
& =(-1)^{r} r ! \int_{c}^{d} \varphi(s) \int_{c}^{s} t^{r} \varphi^{(r)}(t) d t d s \\
& =(-1)^{r} r ! \sum_{i=0}^{r} \frac{(-1)^{i} r !}{(r-i) !} \int_{c}^{d} s^{r-i} \varphi^{(r-i-1)}(s) \varphi(s) d s \\
& =(r !)^{2} \int_{c}^{d} \varphi(s)\left(\int_{c}^{s} \varphi(t) d t\right) d s .
\end{aligned}
$$

Further,

$$
\begin{aligned}
\int_{c}^{d} \varphi(s)\left(\int_{c}^{s} \varphi(t) d t\right) d s & =\int_{c}^{d}\left(\int_{c}^{s} \varphi(t) d t\right) d\left(\int_{c}^{s} \varphi(t) d t\right) \\
& =\left.\frac{1}{2}\left(\int_{c}^{s} \varphi(t) d t\right)^{2}\right|_{c} ^{d}=\frac{1}{2},
\end{aligned}
$$

and $J_{2 r, r}=(r !)^{2} / 2$. So, $I_{k}=0$ for $k=0,1, \ldots, 2 r-1$ and

$$
I_{2 r}=\frac{(2 r) !}{2} B\left(r+\frac{1}{2}, r+\frac{1}{2}\right)=\frac{((2 r-1) ! !)^{2} \pi}{2^{2 r+1}} .
$$

Finally we have

$$
\begin{aligned}
& \left\langle\widetilde{x_{+}^{-r-1 / 2}}\left(\varphi_{\varepsilon}, x\right) \cdot \widetilde{x_{-}^{-r-1 / 2}}\left(\varphi_{\varepsilon}, x\right), \psi(x)\right\rangle \\
& =\frac{\pi}{2} \frac{\psi^{(2 r)}(0)}{(2 r) !}+O(\varepsilon) \\
& =\frac{\pi}{2(2 r) !}\left\langle\delta^{(2 r)}(x), \psi(x)\right\rangle+O(\varepsilon) .
\end{aligned}
$$

Therefore passing to the limit, as $\varepsilon \rightarrow 0$, we obtain (22) proving the theorem.

\section{References}

[1] S. Gasiorawics, Elementary Particle Physics, John Wiley \& Sons, New York, NY, USA, 1966.

[2] M. Oberguggenberger, Multiplication of Distributions and Application to Partial Differential Equations, vol. 259 of Pitman Research Notes in Mathematics Series, Longman, 1992.

[3] H. König, "Neue Begründung der theorie der distributions," Mathematische Nachrichten, vol. 9, pp. 129-148, 1953.

[4] P. Antosik, J. Mikusinski, and R. Sigorski, theory of Distributions. The Sequential Approach, Elsevier, Amsterdam, The Netherlands, 1973. 
[5] B. Fisher, "The product of distributions," The Quarterly Journal of Mathematics, vol. 22, pp. 291-298, 1971.

[6] B. Fisher, "A noncommutative neutrix product of distributions," Mathematische Nachrichten, vol. 108, pp. 117-127, 1982.

[7] L. Z. Cheng and B. Fisher, "Several products of distributions on $\mathbf{R}^{m}$," Proceedings of the Royal Society London A, vol. 426, no. 1871, pp. 425-439, 1989.

[8] A. H. Zemanian, Distribution Theory and Transform Analysis, Dover, 1965.

[9] I. M. Gel'fand and G. E. Shilov, Generalized Functions, vol. 1, chapter 1, Academic Press, New York, NY, USA, 1964.

[10] J.-F. Colombeau, New Generalized Functions and Multiplication of Distributions, vol. 84 of North-Holland Mathematics Studies, North-Holland, Amsterdam, The Netherlands, 1984.

[11] B. Jolevska-Tuneska, A. Takači, and E. Ozcag, "On differential equations with nonstandard coefficients," Applicable Analysis and Discrete Mathematics, vol. 1, no. 1, pp. 276-283, 2007.

[12] B. Damyanov, "Results on Colombeau product of distributions," Commentationes Mathematicae Universitatis Carolinae, vol. 38, no. 4, pp. 627-634, 1997.

[13] B. Damyanov, "Some distributional products of Mikusiński type in the Colombeau algebra $G\left(R^{m}\right)$," Journal of Analysis and its Applications, vol. 20, no. 3, pp. 777-785, 2001.

[14] B. Damyanov, "Modelling and products of singularities in Colombeau's algebra $G(R)$," Journal of Applied Analysis, vol. 14, no. 1, pp. 89-102, 2008.

[15] J. Mikusiński, "On the square of the Dirac delta-distribution," Bulletin de l'Académie Polonaise des Sciences, vol. 14, pp. 511-513, 1966. 


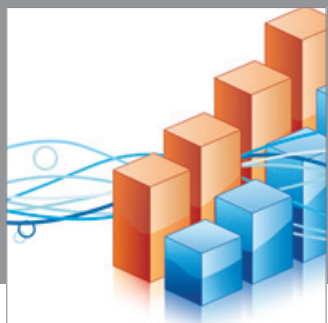

Advances in

Operations Research

mansans

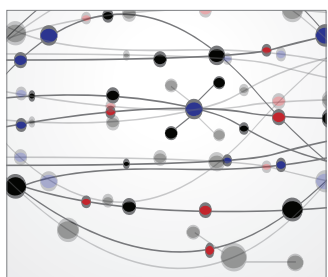

The Scientific World Journal
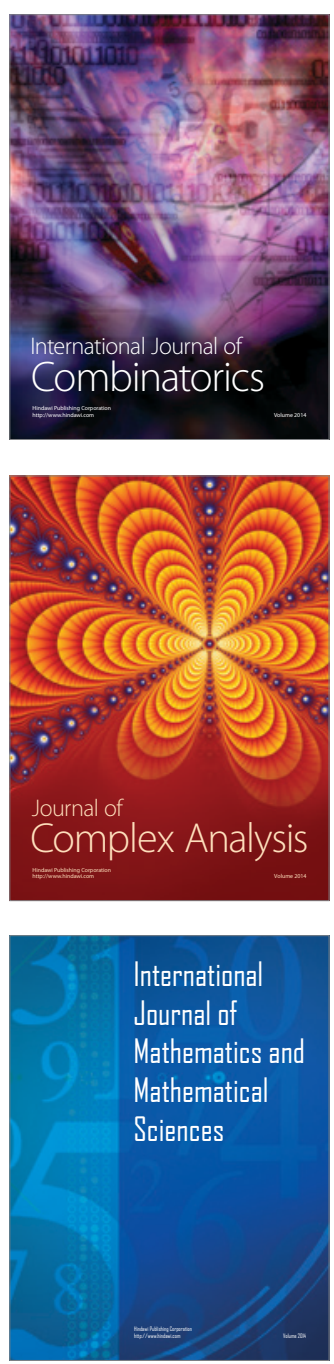
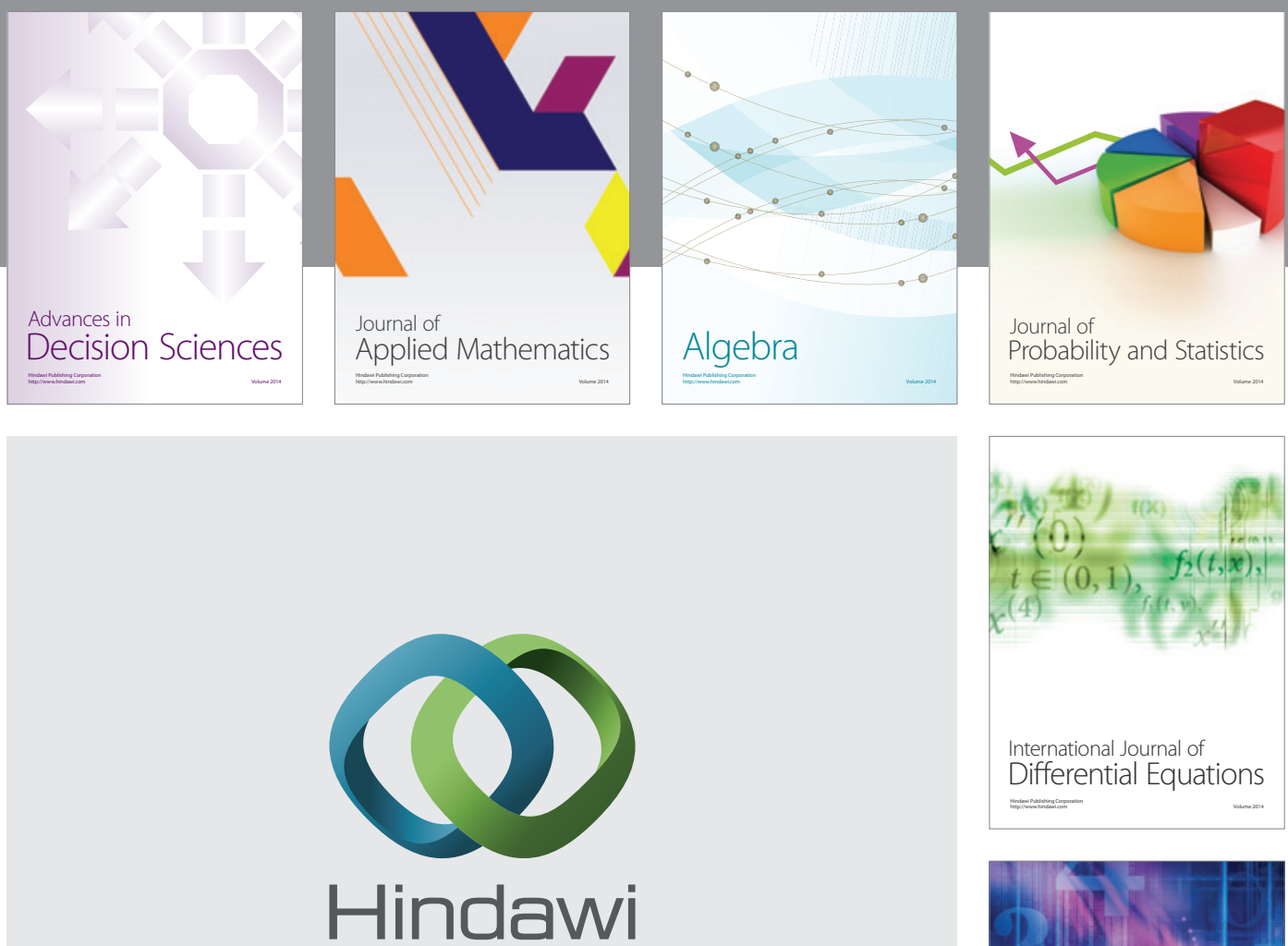

Submit your manuscripts at http://www.hindawi.com
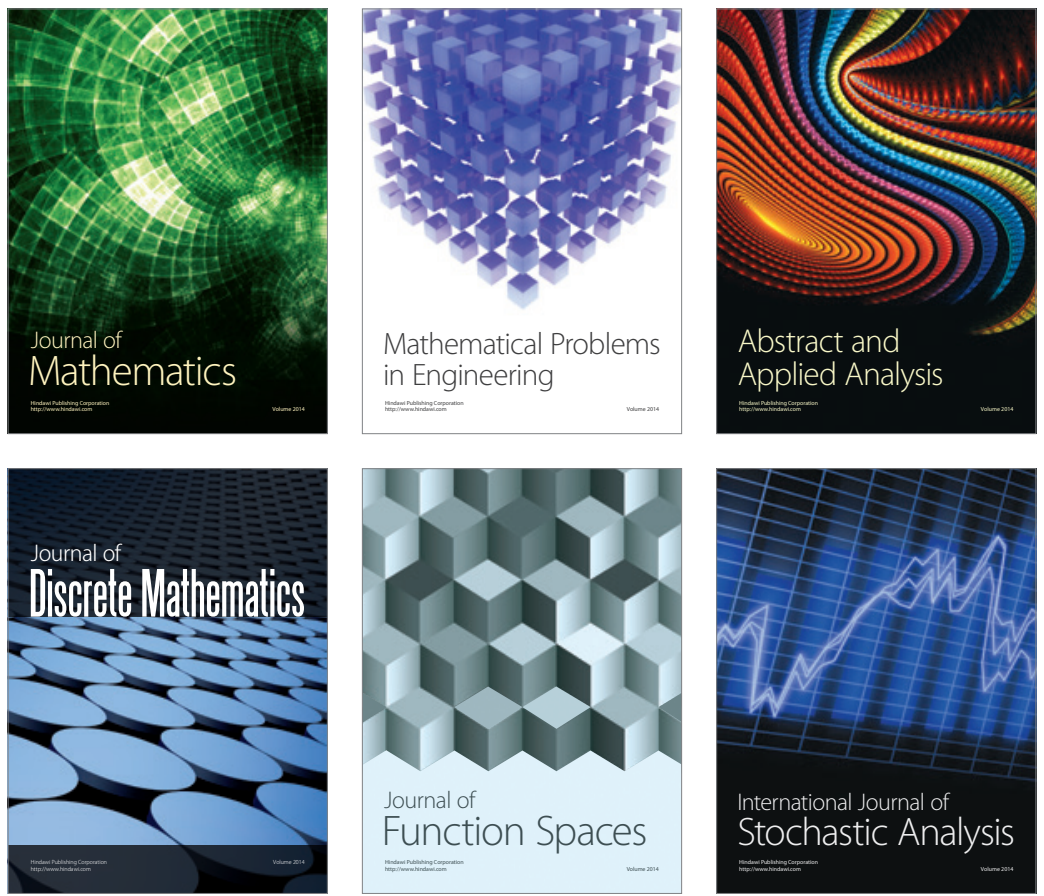

Journal of

Function Spaces

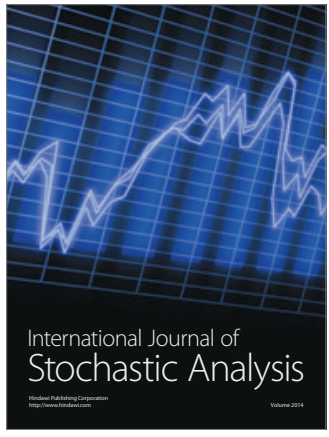

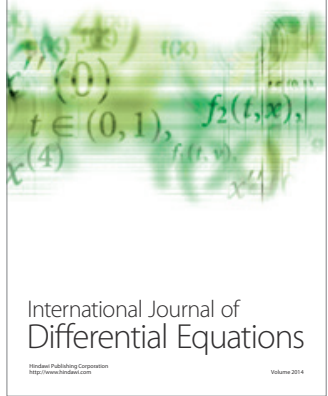
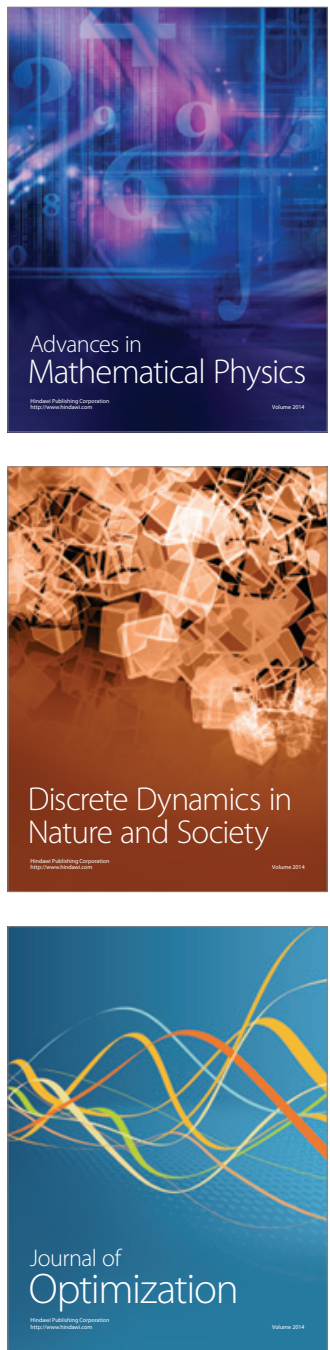